\title{
¿Mejora el torniquete la supervivencia del combatiente en zonas en conflicto?
}

\author{
González Alonso V. ${ }^{1}$, Usero Pérez MC. ${ }^{2}$, Orbañanos Peiro L. ${ }^{3}$, Colmenar Jarillo G. ${ }^{1}$, \\ Gómez Crespo JM. ${ }^{1}$, Hossain López S. ${ }^{1}$
}

Sanid. mil. 2015; 71 (1): 22-28; ISSN: 1887-8571

\begin{abstract}
RESUMEN
Antecedentes: El uso de los torniquetes ha estado presente en los conflictos bélicos como elemento terapéutico para el control de la hemorragia externa en miembros, no obstante, su empleo ha estado rodeado de controversias y supersticiones, a pesar de su probada utilidad. La presente revisión evalúa los beneficios que aporta el torniquete en la atención a los heridos en los modernos conflictos bélicos. El uso pre-hospitalario del torniquete es una medida generalizada y protocolizada en el contexto militar, y está fundamentada en estudios científicos y en una experiencia clínica correspondiente al uso de estos dispositivos en ZO. Objetivos: Analizar mediante la revisión de las referencias encontradas los beneficios y los inconvenientes que ofertan estos dispositivos y si realmente mejoran la supervivencia del combatiente con hemorragia externa en miembros. Material y métodos: Revisión bibliográfica de diversos estudios clínicos efectuados en diversas Zonas de Operaciones (ZO) de los últimos once años. Resultados: el análisis de diversos estudios han demostrado que el uso precoz del torniquete en el campo de batalla, supone una mejora en los ratios de supervivencia y una disminución de la morbimortalidad, del consumo de hemoderivados y por lo tanto, de los costes económicos en el tratamiento de este tipo de heridos. Esta revisión bibliográfica pretende demostrar que el empleo precoz del torniquete en $\mathrm{ZO}$ aumenta la supervivencia de los heridos que presentan hemorragia externa en combate.
\end{abstract}

PALABRAS CLAVE: Torniquete, Control de la hemorragia, Hemostasia, Extremidades, Combate.

\section{Does the tourniquet improve the survival of the fighter in conflict zones?}

SUMMARY: Background: The use of tourniquets has been in the wars as a therapeutic element to control the external bleeding, however, their use has been surrounded by controversy and superstitions, despite its proven utility. This review assesses the benefits of the tourniquet in care of the wounded soldiers in modern warfare. Pre-hospital use of the tourniquet is widespread and notarized as in the military context, and is based on scientific studies and clinical experience for the use of these devices in ZO. Objectives: To analyze by reviewing the references found the benefits and drawbacks that offer these devices and whether it improves survival fighter with external bleeding members. Material and Method: Literature review of several clinical studies in various combat areas of the last eleven years. Results: The analysis of several studies have shown that early use of the tourniquet on the battlefield, an improvement in survival rates and decreased morbidity and mortality, consumption of blood and therefore the economic costs treatment of such injuries. This literature review aims to demonstrate that early use of tourniquet $\mathrm{ZO}$ increases the survival of the casualty who have external bleeding in combat.

KEYWORDS: Tourniquet, Control bleeding, External bleeding, Combat.

\section{INTRODUCCIÓN Y OBJETIVOS}

La actual revisión bibliográfica recoge los últimos estudios clínicos realizados durante los conflictos bélicos más recientes y de mayor transcendencia. En ella, se pretende evaluar si los torniquetes son elementos seguros de tratamiento y que la morbilidad provocada por su empleo, aun siendo relativamente baja, es asumible debido a los beneficios que su uso aporta en el tratamiento de heridos en el campo de batalla (Figura 1 y 2). Los torniquetes tienen una larga y accidentada historia. Desde su in-

\footnotetext{
${ }^{1}$ Cap. Enfermero. Escuela Militar de Sanidad, Dpto. Enfermería. Madrid. España. ${ }^{2}$ Cap. Enfermera. Dirección de Sanidad del ET. Madrid. España.

${ }^{3}$ Cte. Enfermero. Escuela Militar de Sanidad, Dpto de Enfermería. Madrid. España.
}

Dirección para correspondencia: Cap. D. Valentín González Alonso. Escuela Militar de Sanidad. Departamento de Enfermería. Camino de los Ingenieros nº 28047 Madrid. Teléfono: 913647372 / 8147372. Fáx: 913647408.vgonal2@oc.mde.es

Recibido: 28 de octubre de 2013

Aceptado: 27 de marzo de 2014 troducción posiblemente en la antigua Roma hasta el momento presente, su configuración básica se ha modificado muy poco. El primer uso del torniquete para controlar una hemorragia por una lesión traumática no es conocido con absoluta certeza, pero el uso de dispositivos similares ha sido descrito desde la época de los griegos ${ }^{1}$. A lo largo de la historia y asociados al tratamiento de heridos de diferentes campañas bélicas, médicos y cirujanos fueron modelando, perfeccionando dicho dispositivo y definiendo las indicaciones de su empleo en el entorno militar.

Entre las principales causas prevenibles de muerte en combate encontramos la hemorragia externa exanguinante. Ésta se asocia, en la mayoría de los casos, a un trauma vascular severo originado por el empleo de armas de fuego, o bien, por el uso de explosivos de gran potencia, también denominados Improvised Explosive Device (IED). Estos dispositivos al ser detonados a escasa distancia del combatiente, provocan graves lesiones vasculares, con la consecuente pérdida hemática, siendo responsables de un alto porcentaje de bajas en combate en los escenarios bélicos modernos (Figura 3). 


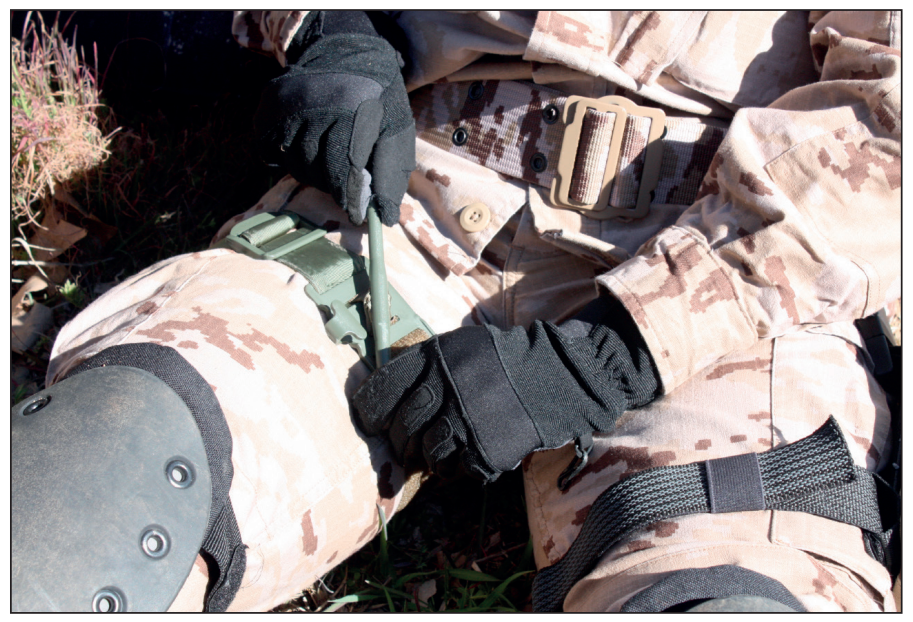

Figura 1. Detalle colocación torniquete proximal a raíz de miembro inferior (Fuente propia).

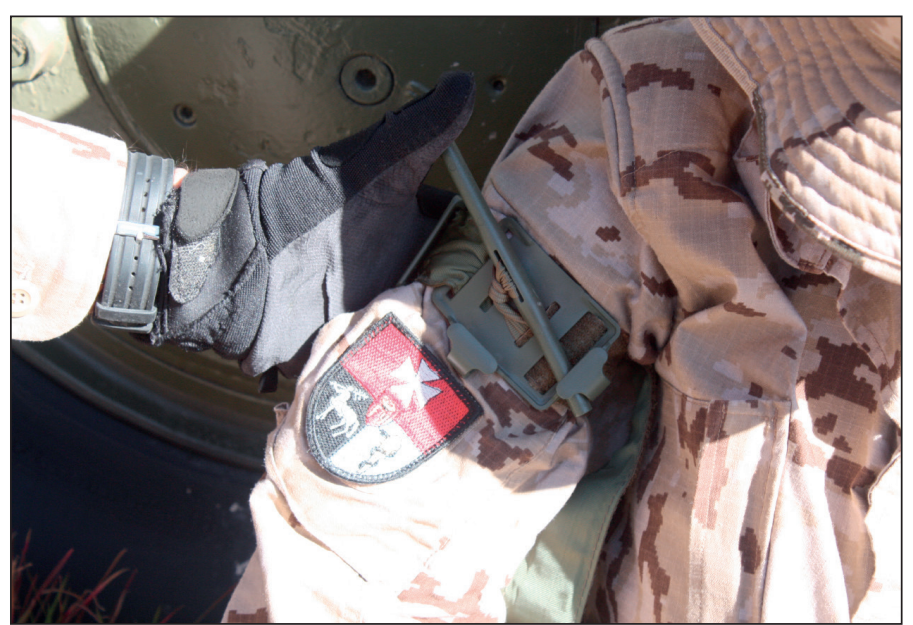

Figura 2. Detalle colocación torniquete a nivel proximal en miembro superior (Fuente propia).

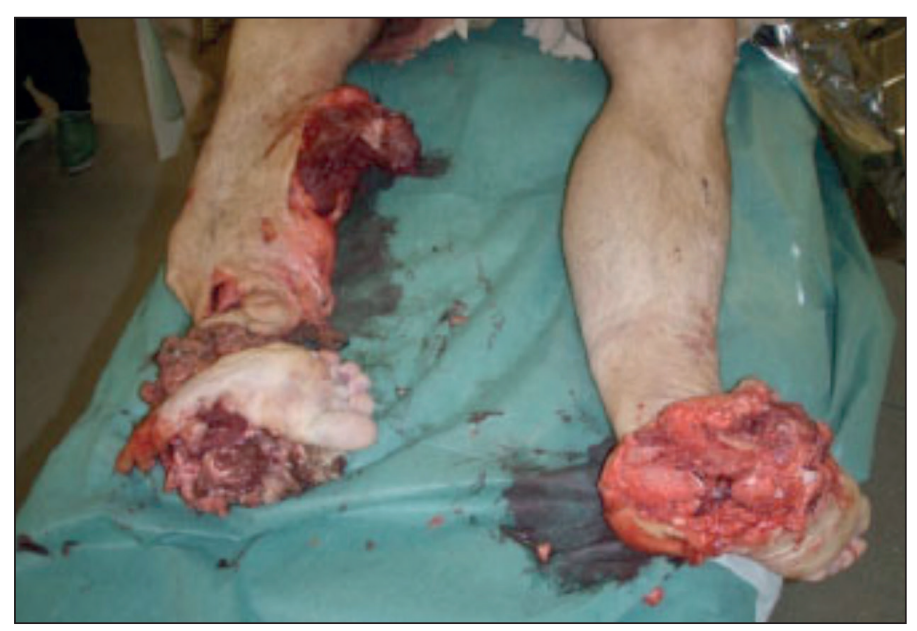

Figura 3. Lesiones provocadas por IED (fuente: http://www.defence.gov.au).
Los esfuerzos de diferentes organizaciones y de la Sanidad Militar de todos los ejércitos, se orientan a controlar de modo precoz la pérdida hemática, para así, disminuir el riesgo de muerte por exanguinación, disminuir la aparición de una coagulopatía de consumo, de acidosis e hipotermia, disminuir el aporte de líquidos en la resucitación y prevenir el shock Hipovolémico. Por todo ello, los torniquetes han constituido un elemento estándar en los equipos de medicina militar. Desde hace unos años, los cuerpos de la Armada y de Infantería de Marina estadounidenses, y más recientemente, las dotaciones de las unidades de las Fuerzas Armadas (FAS) españolas desplegadas en Afganistán, disponen de torniquetes en el botiquín individual del combatiente (BIC), y reciben la formación pertinente para un adecuado empleo del mismo ${ }^{2}$ (Figura 4).

Las actuales tácticas de combate desarrolladas en los modernos conflictos bélicos asimétricos, han incrementado la incidencia de los traumatismos localizados en las extremidades, lo que en combinación con el reconocimiento de que muchas de las muertes por heridas en combate podrían haber sido evitadas con la aplicación de un torniquete para efectuar el control de la hemorragia, constituye una razón más para el uso del torniquete en los actuales conflictos béli$\cos ^{3,4}$. Sebesta, que ha detallado su experiencia como cirujano en un hospital militar de apoyo al combate, señala que «los torniquetes representan un tratamiento esencial, en función de la experiencia más reciente obte-

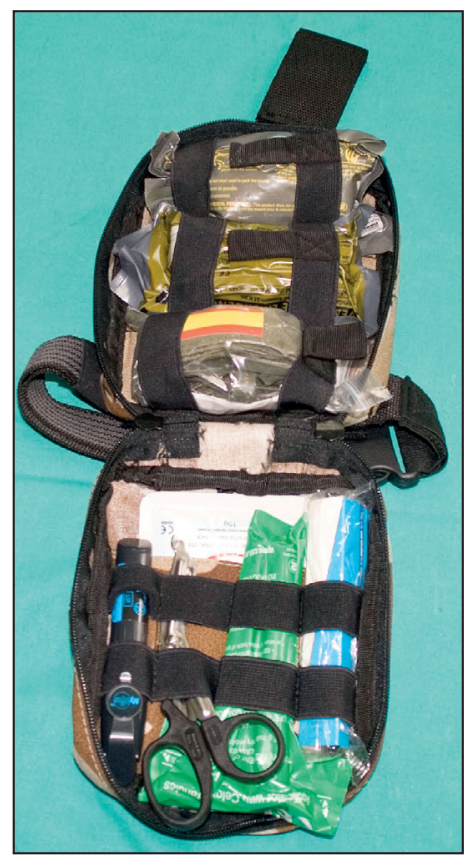

Figura 4. Botiquín individual del combatiente. (Fuente propia). nida en Irak» ${ }^{5}$.

Este fundamento queda reforzado adicionalmente por las circunstancias que influyen en la asistencia sanitaria a los heridos en el campo de batalla. La amenaza real del enemigo, las condiciones ambientales desfavorables, una orografía del terreno difícil, unos tiempos de evacuación más prolongados respecto a la sanidad civil, la austeridad de la logística y la existencia de múltiples heridos en un contexto de capacidades limitadas de clasificación y de tratamiento, son elementos que apoyan el uso oportuno de los torniquetes.

Actualmente, se estima que 7 de cada 100 muertes en combate podrían prevenirse mediante el empleo adecuado de torniquetes $^{6}$. El Comitte of Tactical Combat Casualty Care (CoTCCC) ${ }^{7}$ y el Prehospital Trauma Life Support (PHTLS) ${ }^{8}$, en su edición militar, definen que las principales causas de muerte prevenibles en combate son la hemorragia exanguinante, el neumotórax a tensión y la obstrucción de la vía aérea, por este orden.

Más recientemente, Kelly et $\mathrm{al}^{9}$ publicaron un análisis retrospectivo de los datos obtenidos en las autopsias de los combatien- 
tes en la guerra de Iraq y señalaron los siguientes porcentajes de muertes en el campo de batalla prevenibles:

- Hemorragia externa en miembros $(33 \%)$

- Obstrucción de la vía aérea (10-15\%)

Se considera oportuno pues, el determinar el nivel de eficacia del torniquete en la atención a la baja de combate para poder prevenir una de las principales causas de muerte en el campo de batalla.

Los objetivos de esta revisión son los siguientes:

\section{Objetivo General:}

- Conocer el estado actual del empleo precoz del torniquete para el control de la hemorragia externa en el combatiente que ha sufrido trauma severo en extremidades.

\section{Objetivos específicos:}

- Revisar la bibliografía de los estudios clínicos realizados en diversas zonas de conflicto en los últimos 11 años.

- Valorar la opinión publicada sobre la mejora de la supervivencia tras su uso.

- Valorar la opinión publicada sobre la escasa morbilidad que genera su empleo en el campo de batalla.

- Elaborar una serie de recomendaciones prácticas sobre el empleo del torniquete en operaciones militares.

\section{Interés y relevancia del tema}

Una de las lecciones aprendidas en la última década sobre el tratamiento de los heridos en combate es el empleo, precoz y adecuado, del torniquete para el control de la hemorragia externa cuando un combatiente sufre una lesión hemorrágica por trauma grave en miembros. Tradicionalmente, se han considerado a los torniquetes como una herramienta peligrosa en manos de los profesionales de la asistencia prehospitalaria, contemplándose como último recurso a utilizar en el tratamiento de heridos con hemorragia externa. Su uso en los actuales conflictos bélicos, junto con una rápida evacuación a escalones de asistencia especializada, ha contribuido a salvar gran número de heridos de una muerte segura. Sin embargo su empleo en situaciones de combate ha suscitado un amplio debate en la bibliografía, encontrando opiniones a favor y en contra ${ }^{10-13}$.

A pesar de todo, estos dispositivos han experimentado un incremento espectacular de su popularidad en el último decenio debido a que, en el trascurso de los últimos conflictos bélicos, se ha insistido notablemente en una rápida hemostasia en el propio campo de batalla, ya que la hemorragia constituye una de las principales causas de muerte prevenibles en el campo de batalla ${ }^{14-16}$. Por ello, esta revisión se considera pertinente en el marco de la sanidad militar, y tiene relevancia puesto que los resultados de los estudios analizados aclararán las posibles dudas acerca del empleo de este elemento de tratamiento en el campo de batalla ${ }^{17,18}$.

\section{ANTECEDENTES Y SITUACION ACTUAL}

En 1993 el CoTCCC diseñó un conjunto de protocolos de atención al trauma en combate que fueron publicados como suplemento del manual de Medicina Militar en 199619. Estos protocolos se desarrollaron a partir de la experiencia y de la evidencia obtenida en diversos conflictos bélicos, con el fin de disminuir la aparición de más heridos en combate, completar la misión asignada y salvar el máximo número de vidas disminuyendo la morbilidad de las heridas. La experiencia de los últimos conflictos bélicos ha quedado recogida en las guías del Tactical Combat Casualty Care (TCCC), sometidas a revisiones semestrales para ser actualizadas acorde a las «lecciones aprendidas» sobre el terreno en ZO. Hace solo unos meses, el Gobierno español ratificó el acuerdos de estandarización o Standardization Agreement 2549 (STANAG 2549 (Ed.1) ${ }^{20}$ de la Organización del Tratado del Atlántico Norte (OTAN), donde se recomienda impartir formación según doctrina TCCC al personal que va a ser desplegado en un $\mathrm{TO}^{21}$.

No obstante, y a pesar de los cambios introducidos, según un informe relativo a la guerra de Iraq, la aplicación correcta de un torniquete hubiera permitido evitar más del 50\% de los fallecimientos debidos a una hemorragia aislada en una de las extremidades ${ }^{22}$.

\section{MATERIAL Y MÉTODOS}

\section{Fuentes de información}

Se ha revisado la literatura científica publicada entre 2000 y 2010 en las siguientes bases de datos bibliográficas:

- Medline: de 12 referencias.

- PubMed (NLM): 24 referencias.

- Cochrane Library: 138 referencias.

- Google Academy: 214 referencias.

\section{Metodología de búsqueda}

Se ha utilizado la siguiente metodología de búsqueda en Medline: ((«military personnel»[MeSH Terms] OR («military»[All Fields] AND «personnel»[All Fields]) OR «military personnel»[All Fields] OR «military»[All Fields]) AND («tourniquets»[MeSH Terms] OR «tourniquets»[All Fields] OR «tourniquet»[All Fields])) OR ((«haemorrhage»[All Fields] OR «hemorrhage»[MeSH Terms] OR «hemorrhage»[All Fields]) AND («prevention and control»[Subheading] OR («prevention»[All Fields] AND «control»[All Fields]) OR «prevention and control»[All Fields] OR «control»[All Fields] OR «control groups»[MeSH Terms] OR («control»[All Fields] AND «groups»[All Fields]) OR «control groups»[All Fields]) AND combat[All Fields] AND («environment»[MeSH Terms] OR «environment»[All Fields]) AND («hypovolaemic shock»[All Fields] OR «shock»[MeSH Terms] OR «shock»[All Fields] OR («hypovolemic»[All Fields] AND «shock»[All Fields]) OR «hypovolemic shock»[All Fields]) AND («mortality»[Subheading] OR «mortality»[All Fields] OR «survival»[All Fields] OR 


\section{¿Mejora el torniquete la supervivencia del combatiente en zonas en conflicto?}

«survival»[MeSH Terms])) AND («humans»[MeSH Terms] AND (Meta-Analysis[ptyp] OR Practice Guideline[ptyp] OR Randomized Controlled Trial[ptyp] OR Review[ptyp]) AND (English[lang] OR Spanish[lang]) AND «2002/01/02»[PDat] : «2011/12/30»[PDat])

En PubMed se ha seguido la siguiente metodología de búsqueda: «Military Medicine»[Mesh] AND «tourniquet»[All Fields] AND «combat»[All Fields] AND (hasabstract[text] AND «humans»[MeSH Terms] AND Review[ptyp] AND (English[lang] OR Spanish[lang]) AND («2000»[PDAT]: «2011»[PDAT]))

Las categorías descriptores MesH:

All MeSH Categories

Disciplines and Occupations Category [H]

Health Occupations [H02]

Medicine [H02.403]

> Military Medicine [H02.403.500]

Se han realizado búsquedas en las bases de datos usando las siguientes palabras claves en inglés: "tourniquet», "extremity», «hemorrhage control», "combat», "vascular injury», etc.

\section{Criterios de inclusión y exclusión}

Dentro de los criterios de inclusión del estudio se han seleccionado todos los estudios relacionados con el empleo del torniquete en diversas zonas de operaciones, en inglés y español, de los últimos once años, publicados en revistas médicas y científicas de calidad contrastada y rigor científico, y de ciertos documentos (guías de práctica clínica, estándares, informes,...) publicados por sociedades profesionales, agencias gubernamentales y otras entidades científicas, que proporcionan evidencia científica sobre diversos aspectos relacionados con el control de la hemorragia externa en combate. Se han excluido todos aquellos estudios sobre modelo animal o que no estén relacionados con el tratamiento de la hemorragia por lesiones de combate.

\section{Identificación y selección de estudios relevantes}

Se han recuperado un total de 388 referencias, de las cuales se han desestimado las que no cumplían con los criterios de inclusión, después de analizar el título y resumen de cada una de ellas. Las referencias consideradas como pertinentes por el revisor son las que configuran la bibliografía. Hay que señalar la escasez de estudios relevantes y el bajo número de artículos científicos encontrados, que a nuestro parecer constituye una debilidad importante de este trabajo.

\section{RESULTADOS}

A lo largo de estos últimos once años se han publicado en el marco de revistas científicas de prestigio internacional, diversos estudios que reflejan datos prometedores que pueden confirmar la hipótesis inicial de esta revisión bibliográfica y que a continuación procedemos a resumir.
Las actuales técnicas de combate han originado un modelo lesional que da lugar a una alta incidencia de traumatismos con afectación vascular en extremidades. Esto constituye una de las razones que justifica el uso del torniquete en las condiciones de combate modernas. En las últimas guías europeas sobre el manejo del sangrado tras un trauma severo, se recomienda, con un nivel de evidencia 1C, el empleo del torniquete. Dicho dispositivo es considerado como un método sencillo y eficaz para el control de la hemorragia externa en miembros provocada por traumatismo penetrante, lesiones por «blast» o amputaciones traumáticas ${ }^{23}$.

En su artículo, Lakstein et al (2003) analizan la experiencia de la sanidad militar de las Fuerzas Armadas de Israel. Fueron revisados 550 casos de soldados tratados en una formación sanitaria, de los cuales 91 de ellos fueron tratados con torniquetes. Documentaron un alto porcentaje de casos en los que existía una falta de indicación en la aplicación del torniquete (47\%). Sin embargo se determinaron unas tasas de mortalidad por exanguinación del $0 \%$ y una baja incidencia de complicaciones neurológicas $(5.5 \%)$. Las complicaciones existentes se observaron tras tiempos de isquemia entre 109 y 187 minutos. En el 78\% de los casos el dispositivo aplicado efectuó un efectivo control de la hemorragia ${ }^{24}$.

En el 2004, Beekley et al realizaron un extenso estudio durante la operación Iraquí Freedom sobre 3.444 pacientes, de los cuales 2.000 presentaban lesiones en extremidades y 167 pacientes poseían criterios de ingreso en el estudio. De los 167 pacientes, 67 ingresaron en el $31^{\text {st }}$ Combat Support Hospital con torniquetes colocados (grupo T) y un total de 98 sin ellos (grupo NT). Se observó que no existían diferencias sustanciales a su ingreso en los signos vitales, datos analíticos y requerimientos de sangre. En el grupo T se realizó reconstrucción vascular en el 52.5\% vs $29.9 \%$ del grupo NT y el control de la hemorragia fue efectivo en un $83 \%$ del grupo T vs $60 \%$ del grupo $\mathrm{NT}^{22}$.

Kragh et al en el año 2006 realizaron un estudio sobre 232 pacientes que ingresaron en un hospital de campaña durante la II guerra del Golfo. En dicho estudio observaron que el $97 \%$ de los torniquetes tenían indicación (táctica o clínica) en su aplicación, el tiempo de colocación fue menor de 2 horas en el $91 \%$ de los casos. No se asociaron complicaciones y mucho menos amputaciones asociadas únicamente al uso prolongado del torniquete. El porcentaje de fasciotomías en heridos, con tiempos de isquemia menores de dos horas, fue del $28 \%$ y del $36 \%$ en tiempos de isquemia superiores a dos horas. No se asoció un aumento de la mortalidad con tiempos prolongados de isquemia, pero sí se asoció un aumento de la mortalidad y de la ineficacia del control de sangrado cuando se empleaban torniquetes de circunstancias $^{25,26}$.

Brodie S. et $\mathrm{al}^{27}$ documentan su experiencia sobre los 1375 pacientes tratados en hospitales de campaña británicos en las ZO de Afganistán e Iraq de febrero a septiembre de 2007. E1 5.1 $\%$ fueron tratados con uno o dos torniquetes, al $24 \%$ se le aplicaron 2 ó más torniquetes. El ratio de supervivencia fue del $87 \%$.

Kragh et al realizaron un estudio clínico en un período de un año (2006-2007) en un hospital de apoyo al combate en Bagdad (Iraq). Se documentaron 728 heridos con 953 torniquetes aplicados en extremidades. La aplicación pre-hospitalaria del torniquete fue del $70 \%$, de un $11 \%$ únicamente en la sala de ur- 
gencias y de un 18\% cuando se colocaron torniquetes en el lugar del incidente y a nivel hospitalaria ${ }^{28}$.

Kragh et $\mathrm{al}^{29}$ efectuaron dos estudios posteriores durante el 2008, sobre un total de 499 heridos de combate en un hospital de campaña durante la operación Iraqui Freedom. La supervivencia en ambos estudios fue del $87 \%$, siendo las ratio de supervivencia cuando se aplicaba el torniquete a nivel prehospitalario de un $89 \%$ vs a un $78 \%$ cuando el dispositivo se colocaba en la sala de urgencias. La morbilidad por el empleo de torniquetes fue de un $1.7 \%$ y de un $1.5 \%$ en ambos períodos de estudio respectivamente. También observaron una ratio del $90 \%$ de supervivencia en los pacientes, si el torniquete se aplicaba antes de que se presentara el cuadro de shock y de un $4 \%$ si el torniquete se aplicaba después de que el cuadro de shock estuviera presente.

La mayoría de los torniquetes aplicados en Bagdad (91\%) por períodos de tiempo iguales o inferiores a dos horas presentaron una baja incidencia de complicaciones. Tiempos prolongados de isquemia superiores a 8 horas incrementan las complicaciones, como la miopatía, no obstante, los datos examinados muestran un riesgo limitado ${ }^{25,30}$.

\section{DISCUSIÓN}

El control precoz de la hemorragia está asociado con una pérdida hemática menor, menor necesidad de reposición de hemoderivados en la resucitación del paciente y con un aumento de la supervivencia ${ }^{31}$. El control tardío de la hemorragia está asociado con un aumento de la mortalidad. En la operación Libertad Duradera (Iraq) se demostró que los torniquetes son herramientas efectivas para el control del sangrado en el medio táctico $^{29,31,32}$.

El empleo del torniquete en pacientes sangrantes, permite una resucitación precoz y más efectiva. Además, dilata los tiempos de supervivencia (permitiendo en ese período la llegada de refuerzos humanos y materiales) y proporciona más tiempo para la reanimación y la realización de determinadas técnicas de soporte vital avanzado circulatorio o traumatológico. Así mismo, el empleo del torniquete reduce las necesidades de transfusión y los riesgos de una resucitación agresiva con fluidos, dando unas tasas altas de recuperación de los heridos ${ }^{31,33-35}$.

En vista de los datos aportados por los diversos estudios analizados, en esta revisión bibliográfica, podemos afirmar que factores como la formación y el entrenamiento adecuado del personal que presta la primera asistencia, la doctrina adaptada al medio donde se desarrolla el tratamiento del herido, el correcto empleo de dispositivos diseñados para el control de la hemorragia en los tiempos adecuados, la continua monitorización del paciente y una evacuación rápida hacia un nivel asistencial con capacidad quirúrgica, influyen en el aumento de la supervivencia del combatiente y no se ha asociado a una mayor pérdida de miembros a consecuencia de su uso ${ }^{36,37}$. La aplicación de dispositivos inadecuados, una escasa o inadecuada formación, la inexistencia de doctrina y dilatados tiempos de evacuación, aumenten considerablemente los tiempos de isquemia y han mostrado tasas mayores de morbimortalidad ${ }^{12,38}$.

Sin embargo, debido al escaso número de estudios científicos realizados sobre el tema, se cree precisa la realización de futuros estudios con muestras de población aún más extensas y que examinen la efectividad de varios tipos de dispositivos, los efectos sobre la supervivencia, incidencia de lesión neurológica y de otras complicaciones asociadas con la isquemia en los conflictos bélicos modernos. De este modo y conjuntamente con la creación de una base de datos sobre la etiología de las lesiones de combate en las Fuerzas Armadas españolas, se podría incrementar la evidencia acerca de los beneficios del empleo del torniquete en ambientes tácticos, e incluso en determinadas circunstancias de la emergencia pre-hospitalaria civil. Así, podremos favorecer el desarrollo de una doctrina actualizada en nuestras Fuerzas Armadas, orientar de un modo más realista y efectiva la docencia de nuestro personal sanitario y potenciar con mayor evidencia y seguridad, las indicaciones de uso de este tipo de dispositivos. Todo ello supondrá un incremento en el nivel de recomendación de empleo de dichos elementos, en el ámbito de la asistencia sanitaria pre-hospitalaria militar y civil.

De todo lo anteriormente expuesto se podrían sugerir las siguientes recomendaciones prácticas del uso y empleo del torniquete:

- El objetivo principal de un torniquete es el control del sangrado y la coartación del pulso distal del miembro sobre el que se aplica.

- La formación e instrucción en el manejo y aplicación del torniquete, el entrenamiento y la doctrina de uso deberían ser actualizadas y basadas en la evidencia científica existente.

- Se ha de insistir y dedicar más tiempo a la formación y entrenamiento del «primer interviniente» que va a brindar los cuidados iniciales al herido en ZO.

- Han de emplearse dispositivos diseñados a tal efecto, con unos criterios de diseño científicos, testados previamente y clínicamente validados.

- De los torniquetes evaluados el Combat Application Tourniquet (CAT) es el mejor valorado en el medio prehospitalario y medio táctico y el Emergency and Military Tourniquet (EMT) en el medio hospitalario (sala de urgencias).

- El torniquete si es aplicado antes de evidenciarse signos de shock, aumenta más la tasa de supervivencia que cuando se aplica ante una clínica de shock instaurada. Hay que colocarlo siempre antes de la extracción o de la evacuación del herido.

- La función del miembro sobre el que se ha colocado un torniquete no está asociada a una mayor morbilidad por su uso.

- El torniquete es la única técnica que se aplica en la situación bajo fuego (Care Under Fire), verificando su eficacia en la fase de cuidados tácticos (Tactical Field Care) según la doctrina del TCCC.

- Se recomienda aplicar el torniquete directamente sobre la piel y evitar cubrir éste con un vendaje, siempre que la situación lo permita.

- Si un torniquete es inefectivo aplique otro por encima del mismo, hasta un control efectivo del sangrado.

- Los torniquetes empleados para el control de la hemorragia por trauma severo en miembros inferiores incrementan la supervivencia y permiten una rápida resucitación si 


\section{¿Mejora el torniquete la supervivencia del combatiente en zonas en conflicto?}

dichos dispositivos se han usado siguiendo las indicaciones recomendadas y en los tiempos indicados.

- Que se marque una «T» seguida de la hora a la que se aplica la isquemia, en un lugar visible del paciente, generalmente la frente.

- Los ratios de salvación de miembros son altos cuando son aplicados los principios de control de daño, de resucitación y cirugía vascular.

\section{CONCLUSIONES}

Se ha demostrado que instruir al primer interviniente en materia de primeros auxilios en ambientes tácticos es vital para disminuir las muertes por exanguinación.

Así mismo, el empleo precoz del torniquete y el uso combinado de productos hemostáticos reduce la morbimortalidad de los combatientes como el consumo de hemoderivados en escalones logísticos sanitarios superiores, colaborando en la disminución de los costes y de los tiempos de hospitalización en formaciones sanitarias desplegadas en $\mathrm{ZO}$.

Los porcentajes y el tiempo de supervivencia, junto con los ratios de recuperación de la extremidad son mejores con el uso de torniquetes de dotación y peores cuando se aplican torniquetes de circunstancias o improvisados.

El uso precoz de torniquetes no sólo previene la aparición de shock en dichos pacientes, sino que además, aumenta los índices de supervivencia, sin asociar su uso con una elevada aparición de complicaciones secundarias, y presentando unos niveles de morbilidad más que aceptables. La prevención de las complicaciones sistémicas mediante su uso racional y la elaboración de protocolos estandarizados, con unos tiempos de aplicación reducidos y asegurando una evacuación rápida del herido a un escalón de asistencia sanitaria con capacidad quirúrgica.

Que el torniquete es el elemento de tratamiento de elección para el control de la hemorragia externa moderada o severa en extremidades, sobre todo, en el marco de escenarios tácticos.

Los torniquetes son herramientas útiles para salvar vidas, siempre y cuando estén ligadas a un adecuado entrenamiento del personal, una doctrina moderna en su uso, un diseño acorde a las nuevas tecnologías y dentro del marco de un sistema de atención al trauma con rápida evacuación. Esta combinación ha supuesto un mayor aumento de la tasa de supervivencia en ZO y no se ha asociado una mayor pérdida de miembros debido al correcto uso del torniquete.

La aplicación de dispositivos inadecuados, una escasa o inadecuada formación, la inexistencia de doctrina y dilatados tiempos de evacuación, aumenten considerablemente los tiempos de isquemia y han mostrado tasas mayores de morbimortalidad.

\section{BIBLIOGRAFÍA}

1. LaDran J. The Operations in the Surgery of Mons. London:LaDran; 1749.

2. Noticias Ejército de Tierra. Un botiquín para el combatiente. http://www. ejercito.mde.es/noticias/2010/11/754.html

3. Champion H, Bellamy R, Roberts P, Leppaniemi A. A profile of combat injury. J Trauma 2003;54(5-Suppl):S13-S19.

4. González Alonso V,Colmenar Jarillo G, Rovira Egido JL. Hemorragia en
Campaña: Nuevas Tendencias. Ponencia galardonada con el $2^{\circ}$ accésit en el $6^{\circ}$ Congreso Nacional de Enfermería de la Defensa celebrado en Zaragoza del 30 de septiembre al 3 de octubre de 2008

5. Sebesta J. Special lessons learned from Iraq. Surg Clin North Am.2006;86(3):711-726.

6. Mabry RL, Holcom JB, Baker AM, et al. United States Army Rangers in Somalia: an analysis of combat casualties on an urban battlefield. The Journal of Trauma. 2000;49:515-28 discusion 28-9.

7. Tactical Combat Casualty Care Guidelines 2011. Providers Manual for TCCC.

8. PHTLS: Soporte Vital Básico y Avanzado en el Trauma Prehospitalario. Edición Militar. Ed. Elsevier. 6 $6^{\mathrm{a}}$ Edición revisada. ISBN:978-84-8086-752-B.

9. Kelly JF, Ritenour AE, Mc Laughlin DF, et al. Injury severity and causes of death from Operation Iraqui Freedom ando Operation Enduring Freedom:2003-2004 versus 2006. J. Trauma. 2008;64:S21-27.

10. Navein J, Coupland R, Dunn R. The tourniquet controversy. The Journal of Trauma. 2003;54 5 suppl: S219-20.

11. Husum $\mathrm{H}$ et al. Prehospital tourniquets: there should be no controversy. The Journal of Trauma. 2004; 56:214-5.

12. Pillgram -Larsen J. Compression bandage, not tourniquet: experience in 68 patients with traumatic amputation after mines injuries. RTO HFM Symposium on «Combat casualty Care in Ground Base Tactical Situations: Trauma Technology and emergency Medical Procedures». St. Pete Beach, USA. 16-18 August 2004. Published in RTO-MP-HFM-109.

13. Parker PJ, Clasper J. The Military Tourniquet. JR Army Medical Corps 153 (1): 10-15.

14. Gerard S. Doyle, Peter P. Taillac et al. Los torniquetes: Una revisión de sus indicaciones con propuestas para la ampliación de su uso en el contexto prehospitalario. Prehospital Emergency Care (Ed. Esp.), vol 1, Núm. 4, 2088. 363-379.

15. Holcom J et al. Causes of Death in US Special Operations Forces in the Global War on Terrorism. Annals of Surgery. Volumen 245, number 6, June 2007.

16. Gerhard et al. Out of Hospital Combat Casualty Care. Annals of Emergency Medicine. Vol. 53, no. 2. 2009;169-174.

17. De Lorenzo RA, Porter RS, et al. Tactical Emergency Care: Military and Operational Out of Hospital Medicine. 1999. Ed Brady. ISBN:0-8359-5325-4.

18. Schwartz RB, McManus JC, Swienton RE et al. Tactical Emergency Medicine. Lippincott Williams \& Wilkins. 2008. ISBN-13:978-0-7817-7332-4.

19. Tactical Combat Casualty Care in Special Operations. Military Medicine Supplement 1996. Vol 161. August 1996.

20. http://www.defensa.gob.es/Galerias/info/servicios/ServiciosTecnicos/ST NORM_stanag_implantados.pdf. Documento descargado de la página web el 3/01/2012.

21. STANAG 2549 Ed.1.Emergency Medical Care in the Operational Environment. AMedP-24.

22. Beekley AC, Sebesta JA, Blackbourne LH, et al. Prehospital tourniquet use in Operation Iraqi Freedom: effect on hemorrhage control and outcomes. J Trauma. 2008 Feb;64(2 Suppl):S28-37.

23. Rossaint et al. Management of bleeding following major trauma: an updated European guideline. Critical Care 2010: 14:R52. Descargado de la página web http://ccforum.com/content/14/2/R52.

24. Lakstein D, Blumenfeld A, Sokolov T, et al. Tourniquets for hemorrhage control on the battlefield: a 4 year accumulated experience. J. Trauma 2003 54 (suppl 5): 221-225

25. Kragh JF et al. Practical Use of Emergency Tourniquets to Stop Bleeding in Major Limb Trauma. Journal of Trauma.2008;64:S38-S50.

26. Mabry R, McManus JG. Prehospital Advances in the Management of Severe Penetrating Trauma. Critical Care Medicine 2008;36 (suppl.):S258-S266.

27. Brodie $\mathrm{S}$ et al. Tourniquet Use in combat Trauma: UK Military Experience. Jr Army Medical Corps.153(4):310-313.

28. Kragh JF, O'neill ML, Beebe DF,et al. Survey of the indications for use of emergency tourniquets. Journal of Special Operations Medicine Volume 11, Edition 1 / Winter 11

29. Kragh JF Jr, Littrel ML, Jones JA, Walters TJ, Baer DG, Wade CE, Holcomb JB. Battle casualty survival with emergency tourniquet use to stop limb bleeding. J Emerg Med. 2011 Dec;41(6):590-7. Epub 2009 Aug 31.

30. Tien HC, Jung V, Rizoli SB, et al. An Evaluation of tactical combat casualty care interventions in a combat environment. J Am Coll Surg 2008; 207(2): 174-178. 
31. Artz CP, Howard JM, Sako Y, et al. Clinical experiences in the early management of the most severely injured battle casualties. Ann Surg 1955;141(3):285-96.

32. Owens BD, Kragh JF Jr, Macaitis J, et al. Characterization of extremity wounds in Operation Iraqi Freedom and Operation Enduring Freedom. J Orthop Trauma 2007;21(4):254-7.

33. Fox CJ, Gillespie DL, Cox ED, et al. Damage control resuscitation for vascular surgery in a combat support hospital. J Trauma 2008;65(1):1-9.

34. Fox CJ, Gillespie DL, Cox ED, et al. The effectiveness of a damage control resuscitation strategy for vascular injury in a combat support hospital: results of a case control study. J Trauma 2008;64(Suppl 2):S99-106.
35. Garulo J. La formación en primeros auxilios es clave. Revista Atenea. 2011;(30):89-90.

36. Kragh JF, Baer DG, Walters TJ. Extended (16 hour) tourniquet application after combat wounds: a case report and review of the current literature. J. Orthop. Trauma. 2007;21: 274-278.

37. González Alonso V et al. Control de la Hemorragia Externa en Combate. Prehospital Emergency Care.(Ed. Esp.) vol 2, núm 4, 2009; 293-304.

38. Pillgram-Larsem J, Mellesmo S. Not a tourniquet, but compressive dressing. Experience from 68 traumatic amputations after injuries from mines. English abstract. Tidsskr Nor Laegeforen 1992;112 (17):2188-90. 